\title{
Ipilimumab-Induced Hypophysitis and Uveitis in a Patient With Metastatic Melanoma and a History of Ipilimumab-Induced Skin Rash
}

\author{
Neelima N. Nallapaneni, MBBS, ${ }^{a, *}$; Rajesh Mourya, MBBS ${ }^{b, *} ;$ Vijaya Raj Bhatt, MBBSc; \\ Sakshi Malhotra, MBBS ${ }^{\text {; }}$ Apar Kishor Ganti, MD, MS ; and Ketki K. Tendulkar, MBBS ${ }^{a}$
}

\begin{abstract}
Ipilimumab, a monoclonal antibody that blocks cytotoxic Tlymphocyte-associated antigen-4, leading to enhanced T-cell activation and proliferation, is associated with improved overall survival in melanoma. Its use can result in immune-related adverse events, the most common of which are skin rash, diarrhea, and colitis. Ipilimumab-induced hypophysitis is uncommon, mostly involves anterior pituitary, and is associated with abnormalities in pituitary MRI, whereas uveitis has been rarely reported. These immunerelated adverse events occur during therapy. This report describes a patient who developed uveitis and hypophysitis involving both anterior and posterior pituitary, without MRI findings more than 3 weeks after the fourth dose of ipilimumab. This case illustrates the unusual presentation of and diagnostic challenges associated with ipilimumab-induced immune-related adverse events. (J Natl Compr Canc Netw 2014;12:1077-1081)
\end{abstract}

\section{NCCN: Continuing Education}

\section{Accreditation Statement}

This activity has been designated to meet the educational needs of physicians and nurses involved in the management of patients with cancer. There is no fee for this article. No commercial support was received for this article. The National Comprehensive Cancer Network (NCCN) is accredited by the ACCME to provide continuing medical education for physicians.

NCCN designates this journal-based CME activity for a maximum of 1.0 AMA PRA Category 1 Credit(s) ${ }^{\mathrm{TM}}$. Physicians should claim only

From the aUniversity of Nebraska Medical Center, Department of Internal Medicine, Division of Nephrology; ' ${ }^{\circ}$ Creighton University Medical Center, Department of Internal Medicine; 'University of Nebraska Medical Center Department of Internal Medicine, Division of Hematology and Oncology: ¿VA Nebraska-Western lowa Health Care System, Department of Internal Medicine; and eVA Nebraska-Western lowa Health Care System, and University of Nebraska Medical Center, Department of Internal Medicine, Division of Hematology and Oncology, Omaha, Nebraska.

*These authors contributed equally.

Submitted September 19, 2013; accepted for publication January 27, 2014.

Dr. Ganti has disclosed that he received grant/research support from Pfizer Inc.; NewLink Genetics, Inc.; Astex Pharmaceuticals, Inc.; Amgen Inc.; Boehringer Ingelheim $\mathrm{GmbH}$; and Bristol-Myers Squibb Company, and is a scientific advisor for Otsuka Pharmaceutical Co., Ltd. Dr. Tendulkar has disclosed that her spouse receives grant/research support from Pfizer Inc.; NewLink Genetics, Inc.; Astex Pharmaceuticals, Inc.; Amgen Inc.; Boehringer Ingelheim $\mathrm{GmbH}$; and Bristol-Myers Squibb Company, and is a scientific advisor for Otsuka Pharmaceutical Co., Ltd. The remaining authors have disclosed that they have no financial interests, arrangements, affiliations, or commercial interests with the manufacturers of any products discussed in this article or their competitors. the credit commensurate with the extent of their participation in the activity.

NCCN is accredited as a provider of continuing nursing education by the American Nurses Credentialing Center's Commission on Accreditation.

This activity is accredited for 1.0 contact hours. Accreditation as a provider refers to recognition of educational activities only; accredited status does not imply endorsement by NCCN or ANCC of any commercial products discussed/displayed in conjunction with the educational activity. Kristina M. Gregory, RN, MSN, OCN, is our nurse planner for this educational activity.

All clinicians completing this activity will be issued a certificate of participation. To participate in this journal CE activity: 1) review the learning objectives and author disclosures; 2) study the education content; 3) take the posttest with a $66 \%$ minimum passing score and complete the evaluation at http://education.nccn.org/ node/50873; and 4) view/print certificate.

Release date: August 8, 2014; Expiration date: August 8, 2015

\section{Learning Objectives}

Upon completion of this activity, participants will be able to:

- Recognize the typical timing pattern for the most frequently reported immune-related adverse events associated with ipilimumab

- Describe the unusual presentation of rare complications associated with ipilimumab such as hypophysitis and uveitis

Correspondence: Apar Kishor Ganti, MD, MS, Division of Oncology and Hematology, Department of Internal Medicine, University of Nebraska Medical Center, 987680 Nebraska Medical Center, Omaha, NE 681987680. E-mail: aganti@unmc.edu

EDITOR

Kerrin M. Green, MA, Assistant Managing Editor, JNCCN—Journal of the National Comprehensive Cancer Network

Ms. Green has disclosed that she has no relevant financial relationships.

\section{CE AUTHORS}

Deborah J. Moonan, RN, BSN, Director, Continuing Education \& Grants Ms. Moonan has disclosed that she has no relevant financial relationships.

Ann Gianola, MA, Manager, Continuing Education \& Grants Ms. Gianola has disclosed that she has no relevant financial relationships. Kristina M. Gregory, RN, MSN, OCN, Vice President, Clinical Information Operations

Ms. Gregory has disclosed that she has no relevant financial relationships. 
Ipilimumab is a recombinant fully human monoclonal immunoglobulin that blocks cytotoxic T-lymphocyteassociated antigen-4, leading to tumor cell destruction via enhanced T-cell activation and proliferation. Its use in malignant melanoma is associated with improved overall survival compared with traditional therapies ${ }^{1,2}$; however, activation of CD4 and CD8 T cells and release of inflammatory cytokines can also result in several immune related adverse events ${ }^{3}$ (Table 1). These adverse events, which can affect approximately twothirds of patient, are an important source of morbidity and mortality. The most common immune-related adverse events are skin rash, diarrhea, and colitis. ${ }^{1,3,4}$ Hypophysitis, although one of the most common endocrinopathies, is relatively uncommon with ipilimum$\mathrm{ab}^{1,3,4}$ and can be potentially difficult to diagnose. These immune-related adverse events frequently occur during therapy $^{1}$ and often with a typical timing pattern. ${ }^{3} \mathrm{Al}$ though not universally true, frequently skin rash occurs after 2 to 3 weeks of therapy, diarrhea and colitis after 6 to 7 weeks, and endocrinopathies after approximately 9 weeks. ${ }^{3}$ This report describes a patient who developed hypophysitis and uveitis more than 3 weeks after the fourth dose of ipilimumab, thus highlighting the associated diagnostic challenges.

\begin{tabular}{|ll|}
\hline \multicolumn{2}{|c|}{ Table 1 Ipilimumab-Induced Immune- } \\
Related Adverse Events
\end{tabular}

a Based on pivotal phase III trials on ipilimumab \pm glycoprotein 100 peptide vaccine, ${ }^{1}$ and ipilimumab plus dacarbazine. ${ }^{5}$

${ }^{\mathrm{b}} \mathrm{A}$ high frequency of hepatotoxicity, particularly elevation of liver enzymes, was noted in one study that combined ipilimumab with dacarbazine. ${ }^{5}$ In general, the incidence of immune-related hepatotoxicity reported in the literature is $<5 \%-10 \%$. $^{3}$

\section{Case Report}

A 62-year-old man presented to the emergency department with nausea and vomiting for 5 days and dizziness for 2 days. His past medical history was significant for BRAF V600E mutation-positive melanoma of skin involving left anterior chest wall, pleura, and inguinal lymph node, which was diagnosed 2 years before his presentation to the emergency room. When the disease progressed after therapy with vemurafenib and a high dose of interleukin-2, the patient was started on ipilimumab $(3.0 \mathrm{mg} / \mathrm{kg}$ every 3 weeks for 4 doses). After the first dose of ipilimumab, the patient developed persistent grade 2 erythematous maculopapular skin rash and itching, which was treated with methylprednisone (initial dose, $\approx 0.4 \mathrm{mg} / \mathrm{kg} / \mathrm{d}$ of prednisone equivalent) and led to subsequent dose delays. With subsequent cycles, the patient experienced flares of skin rash requiring low-dose steroid therapy. The patient received the last dose of methylprednisone $(4.0 \mathrm{mg} / \mathrm{d})$ approximately 2 weeks before the presentation. The patient received a total of 4 cycles of ipilimumab, with the last dose given 23 days before presentation.

Examination revealed a blood pressure of 107/74 $\mathrm{mm} \mathrm{Hg}$, heart rate of 52 beats per minute, temperature of $98.5^{\circ} \mathrm{F}$, and respiratory rate of 16 breaths per minute. Physical examination was unremarkable. Laboratory tests revealed the following levels: sodium, $119 \mathrm{mmol} / \mathrm{L}$; potassium, $3.8 \mathrm{mmol} / \mathrm{L}$; chloride, $89 \mathrm{mmol} / \mathrm{L}$; bicarbonate, $25 \mathrm{mmol} / \mathrm{L}$; blood urea nitrogen, $9 \mathrm{mmol} / \mathrm{L}$; and creatinine, $0.9 \mathrm{mg} / \mathrm{dL}$, as well as a serum osmolality of $252 \mathrm{mOsm} / \mathrm{kg}$, and normal hemogram results. Initially, normal saline infusion was started for presumed hypovolemic hyponatremia. Urine sodium and osmolality, measured after saline infusion was started, were found to be $134 \mathrm{mmol} / \mathrm{L}$ and $301 \mathrm{mOsm} / \mathrm{kg}$, respectively. At this point, a diagnosis of syndrome of inappropriate antidiuretic hormone (SIADH) was considered; normal saline infusion was discontinued, one dose of demeclocycline was administered, and the patient was fluidrestricted. Because of the use of ipilimumab, adrenal insufficiency from hypophysitis versus primary adrenal insufficiency was suspected and the patient was started on hydrocortisone (initial dose, $\approx 1 \mathrm{mg} / \mathrm{kg} / \mathrm{d}$ prednisone equivalent), and improvement was seen in his sodium levels.

Further testing revealed levels of thyroid stimulating hormone (TSH) less than $0.02 \mathrm{mIU} / \mathrm{L}$, random 
cortisol less than $0.2 \mathrm{mcg} / \mathrm{dL}$, adrenocorticotropin hormone less than $5 \mathrm{pg} / \mathrm{mL}$, luteinizing hormone less than $0.3 \mathrm{mIU} / \mathrm{L}$, follicle-stimulating hormone less than $0.9 \mathrm{mIU} / \mathrm{mL}$, insulin-like growth factor-1 less than $16 \mathrm{ng} / \mathrm{mL}$, and prolactin of $1.4 \mathrm{ng} / \mathrm{mL}$. A brain MRI revealed a normal pituitary with no evidence of brain metastasis. A CT scan of the abdomen performed to rule out metastasis showed normal adrenal glands bilaterally. In light of these results, a diagnosis of ipilimumab-induced hypophysitis leading to hypopituitarism and hyponatremia was made.

The patient was treated with hydrocortisone replacement, leading to symptom resolution and serum sodium level normalization. During the hospital stay, he complained of excessive thirst and urination, leading practitioners to consider the possibility of a mineralocorticoid effect of hydrocortisone versus diabetes insipidus. The patient developed steroidinduced hallucinations and confusion, and hence hydrocortisone was changed to medium-dose prednisone. He also complained of seeing floaters. On day 5, slit-lamp examination revealed anterior uveitis, for which he was started on prednisone acetate eye drops. The patient was discharged home with oral prednisone and prednisone acetate eye drops on day 6 of hospitalization.

On 2-week follow-up, the anterior uveitis had resolved but he continued to have excessive thirst and urination. He was found to have persistent hypothyroidism, with a TSH level of $0.74 \mathrm{mIU} / \mathrm{L}$ and free thyroxine (fT4) level of $0.4 \mathrm{ng} / \mathrm{dL}$, and was started on thyroxine. A water-deprivation test confirmed the presence of partial diabetes insipidus, and he was started on desmopressin.

Four months after hospitalization, the patient still had occasional dizziness and nausea. He was maintained on levothyroxine, low-dose steroids, and desmopressin. Because he was not symptomatic from hypogonadism, this was being monitored. The patient had a partial response to ipilimumab in his pleural lesion.

\section{Discussion}

In 2 large phase III studies of ipilimumab, hypophysitis was seen in $1.5 \%$ or less of patients treated with ipilimumab. ${ }^{1,5}$ The incidence of immune-related adverse events, ${ }^{3}$ including hypophysitis, ${ }^{6}$ might be dosedependent, with an incidence of $5 \%$ to $17 \%$ seen with a dose exceeding $3 \mathrm{mg} / \mathrm{kg} .{ }^{6}$ However, in a large phase
III study among patients receiving ipilimumab at $10 \mathrm{mg} / \mathrm{kg}$ and dacarbazine $(\mathrm{n}=250)$, only 1 patient developed hypophysitis during maintenance therapy, which was observed more than 70 days after the last dose of ipilimumab. ${ }^{5}$ Although this might indicate that hypophysitis is not dose-dependent, it has also been speculated that the decrease in hypophysitis in these circumstances is related to the use of cytotoxic chemotherapy and radiotherapy, which may prevent ipilimumab-induced hypophysitis by depleting immune cells. ${ }^{6}$ Uveitis is a rare complication of ipilimumab, with only a handful of cases reported in the literature. No case of uveitis was reported in several major trials of ipilimumab., ${ }^{1,7-10}$ Two instances of uveitis have been reported in association with ipilimumab and glycoprotein 100 melanocyte/melanoma differentiation antigen vaccine. ${ }^{11}$

The present patient developed a skin rash after a single dose of ipilimumab, and subsequently developed ipilimumab-induced hypophysitis and uveitis 3 weeks after the last dose. Because the plasma half-life of ipilimumab is 12 to 14 days, ${ }^{3}$ delayed onset of hypophysitis and uveitis is possible. ${ }^{5}$ The use of steroids for the treatment of skin rash could also have masked the onset of hypophysitis and uveitis. Furthermore, as demonstrated by this case, patients with 1 ipilimumab-induced immune-related side effect are at an increased subsequent risk for other immune-related side effects should further doses of ipilimumab be administered.

The clinical presentation of ipilimumabinduced hypophysitis can be diverse, ranging from nausea, vomiting, and hyponatremia to neurologic symptoms, such as ataxia and diplopia. The vague and protean manifestations can present a diagnostic challenge. These symptoms occur after an average of 6 weeks of therapy. ${ }^{3}$ A high index of suspicion is needed to avoid delay in diagnosis and misdiagnosis. Pituitary function assessment through hormone level measurements and MRI of the pituitary are the most useful methods to establish diagnosis. Pituitary MRI can show diffuse enlargement of the pituitary gland with suprasellar extension or uncommonly empty sella ${ }^{12}$; however, as illustrated by this case, results can be essentially normal, ${ }^{13}$ such as in patients receiving steroids or when diagnosed early. When present, pituitary swelling on MRI precedes hormone deficiency. ${ }^{14}$ An instance of intense uptake in the pituitary gland seen on PET/CT scan, before the initiation of hypopituitarism, has been reported, 
suggesting that hypophysitis can be detected early on a PET/CT scan performed for another indication. ${ }^{15}$ Hypophysitis results in anterior pituitary dysfunction, whereas posterior pituitary function often remains normal. Investigators have speculated that corticotroph and possibly thyrotroph cells may be preferentially damaged..$^{13}$ However, few instances of posterior pituitary dysfunction, as in this patient, have also been reported. ${ }^{16,17}$ The most important differential diagnosis of ipilimumab-induced hypophysitis includes brain metastasis from melanoma, primary adrenal insufficiency, and thyroiditis. ${ }^{3}$

Treatment depends on the grade of toxicity. The accepted treatment of symptomatic hypophysitis includes the initiation of a high-dose steroid $(\geq 1 \mathrm{mg} / \mathrm{kg}$ of prednisone or equivalent) with a slow taper over at least 4 weeks, supportive care, and appropriate hormone replacement as indicated. ${ }^{12,18}$ An initial high dose of methylprednisolone (1-2 $\mathrm{mg} / \mathrm{kg}$ ) may be considered, particularly for grade $3 / 4$ toxicities. Grade 2 endocrinopathies can be treated with further doses of ipilimumab in selected cases; however, grade $3 / 4$ toxicities (except grade 3 skin toxicities) often require permanent discontinuation. ${ }^{3}$ Fortunately, steroid use does not seem to reduce the antineoplastic effect of ipilimumab. ${ }^{6}$

The prognosis of hypophysitis is variable, ranging from asymptomatic detection, to spontaneous resolution, to progression to pituitary fibrosis and permanent pan-hypopituitarism. ${ }^{12}$ With steroid use, although symptom resolution can occur within a few days, the size of the pituitary gland gradually declines in approximately 4 to 12 weeks. ${ }^{6}$ In certain cases, the pituitary function may be perturbed for even longer, thus requiring lengthy physiologic hydrocortisone replacement therapy (mean, 20 weeks). ${ }^{3,6}$ Despite the long recovery time, the pituitary thyroid and gonadal axes may recover in up to half of the patients, whereas most of the patients have persistent secondary adrenal insufficiency. ${ }^{6}$ Given the rarity of ipilimumab-induced uveitis, a paucity of information exists on its presentation, diagnosis, or management; however, findings can be expected to be similar to those for uveitis from any other cause. For management, topical steroids for grade $1 / 2$ toxicities and systemic steroids for grade $3 / 4$ uveitis are recommended. ${ }^{3}$

The occurrence of severe immune-related adverse events may be associated with rates of disease control ${ }^{8}$ or durable objective response. ${ }^{19}$ The present patient did not have evidence of disease progression at 6 months.
Although immune-related endocrinopathies cannot be prevented completely, periodic hormonal assays and chemistry profile at baseline and before each dose may be useful for early detection, ${ }^{3}$ as can PET scan performed for restaging. ${ }^{15}$ Close observation for these side effects is particularly important for patients with prior history of immune-related adverse effects.

\section{Conclusions}

Ipilimumab is a novel immunotherapy that provides a survival advantage when used in the treatment of metastatic melanoma. With its increasing use, the possibility exists for the emergence of rare yet serious complications, such as hypophysitis. As illustrated by the present case, ipilimumab can be associated with hypophysitis, which can also involve the posterior pituitary. Normal imaging studies do not always exclude the presence of hypophysitis, and therefore a high index of suspicion, early recognition based on symptoms, and treatment with steroids are important.

\section{References}

1. Hodi FS, O'Day SJ, McDermott DF, et al. Improved survival with ipilimumab in patients with metastatic melanoma. N Engl J Med 2010;363:711-723.

2. Dequen P, Lorigan P, Jansen JP, et al. Systematic review and network meta-analysis of overall survival comparing $3 \mathrm{mg} / \mathrm{kg}$ ipilimumab with alternative therapies in the management of pretreated patients with unresectable stage III or IV melanoma. Oncologist 2012;17:1376-1385.

3. Weber JS, Kahler KC, Hauschild A. Management of immunerelated adverse events and kinetics of response with ipilimumab. J Clin Oncol 2012;30:2691-2697.

4. Weber JS, Dummer R, de Pril V, et al. Patterns of onset and resolution of immune-related adverse events of special interest with ipilimumab: detailed safety analysis from a phase 3 trial in patients with advanced melanoma. Cancer 2013;119:1675-1682.

5. Robert C, Thomas L, Bondarenko I, et al. Ipilimumab plus dacarbazine for previously untreated metastatic melanoma. N Engl J Med 2011;364:2517-2526.

6. Corsello SM, Barnabei A, Marchetti P, et al. Endocrine side effects induced by immune checkpoint inhibitors. J Clin Endocrinol Metab 2013;98:1361-1375.

7. Hersh EM, O'Day SJ, Powderly J, et al. A phase II multicenter study of ipilimumab with or without dacarbazine in chemotherapynaive patients with advanced melanoma. Invest New Drugs 2011;29:489-498.

8. Weber JS, O'Day S, Urba W, et al. Phase I/II study of ipilimumab for patients with metastatic melanoma. J Clin Oncol 2008;26:5950-5956.

9. Margolin K, Ernstoff MS, Hamid O, et al. Ipilimumab in patients with melanoma and brain metastases: an open-label, phase 2 trial. Lancet Oncol 2012;13:459-465. 
Ipilimumab-Induced Hypophysitis and Uveitis

10. Wolchok JD, Neyns B, Linette G, et al. Ipilimumab monotherapy in patients with pretreated advanced melanoma: a randomised, double-blind, multicentre, phase 2, dose-ranging study. Lancet Oncol 2010;11:155-164.

11. Robinson MR, Chan CC, Yang JC, et al. Cytotoxic T lymphocyte-associated antigen 4 blockade in patients with metastatic melanoma: a new cause of uveitis. J Immunother 2004;27:478-479.

12. Blansfield JA, Beck KE, Tran K, et al. Cytotoxic T-lymphocyteassociated antigen-4 blockage can induce autoimmune hypophysitis in patients with metastatic melanoma and renal cancer. J Immunother 2005;28:593-598.

13. Min L, Vaidya A, Becker C. Association of ipilimumab therapy for advanced melanoma with secondary adrenal insufficiency: a case series. Endocr Pract 2012;18:351-355.

14. Yang JC, Hughes M, Kammula U, et al. Ipilimumab (antiCTLA4 antibody) causes regression of metastatic renal cell cancer associated with enteritis and hypophysitis. J Immunother 2007;30:825-830.
15. van der Hiel B, Blank CU, Haanen JB, Stokkel MP. Detection of early onset of hypophysitis by (18)F-FDG PET-CT in a patient with advanced stage melanoma treated with ipilimumab. Clin Nucl Med 2013;38:e182-184.

16. Juszczak A, Gupta A, Karavitaki N, et al. Ipilimumab: a novel immunomodulating therapy causing autoimmune hypophysitis: a case report and review. Eur J Endocrinol 2012;167:1-5.

17. Dillard T, Yedinak CG, Alumkal J, Fleseriu M. Anti-CTLA-4 antibody therapy associated autoimmune hypophysitis: serious immune related adverse events across a spectrum of cancer subtypes. Pituitary 2010;13:29-38.

18. Andrews $S$, Holden R. Characteristics and management of immune-related adverse effects associated with ipilimumab, a new immunotherapy for metastatic melanoma. Cancer Manag Res 2012;4:299-307.

19. Downey SG, Klapper JA, Smith FO, et al. Prognostic factors related to clinical response in patients with metastatic melanoma treated by CTL-associated antigen-4 blockade. Clin Cancer Res 2007;13:6681-6688.

\section{Instructions for Completion}

To participate in this journal CE activity: 1) review the learning objectives and author disclosures; 2 ) study the education content; 3 ) take the posttest with a $66 \%$ minimum passing score and complete the evaluation at http://education.nccn.org/ node/50873; and 4) view/print certificate. After reading the article, you should be able to answer the following multiple- choice questions. Credit cannot be obtained for tests completed on paper. You must be a registered user on NCCN.org. If you are not registered on NCCN.org, click on "New Member? Sign up here" link on the left hand side of the Web site to register. Only one answer is correct for each question. Once you successfully answer all posttest questions you will be able to view and/or print your certificate. Software requirements: Internet.

\section{Posttest Questions}

1. Which of the following statement about ipilimumab use in melanoma is NOT true?

a. Its use has been associated with improved overall survival.

b. It is useful in patients with or without BRAF V600E mutation.

c. It can be associated with adverse events several weeks after the completion of therapy.

d. Steroid use can reduce the antineoplastic effect of ipilimumab.
2. True or False: Blockade of cytotoxic T-lymphocyte-associated antigen-4 with ipilimumab can result in adverse events such as skin rash, colitis, and hypophysitis.

3. True or False: An initial high dose of steroid followed by a quick taper over 1 to 2 weeks is recommended for the management of grade 3-4 immune-related adverse events associated with the use of ipilimumab. 